\title{
Inelastic Deformations of Rocks and their Influence on Development of the Oil and Gas Fields
}

\author{
Guliyev A $^{1}$, Hasanov ${ }^{2}$, Kazymov B $^{1}$, Efendiyev $\mathbf{R}^{1}$, Zeynalov $\mathbf{A}^{2}$ and \\ Smirnova $\mathrm{A}^{3 *}$ \\ ${ }^{1}$ Oil and Gas Institute of the Azerbaijan National Academy of Sciences, Azerbaijan \\ ${ }^{2}$ Azerbaijan State University of Oil and Industry, Azerbaijan \\ ${ }^{3}$ South Dakota School of Mines and Technology, USA
}

*Corresponding author: Alevtina Smirnova, South Dakota School of Mines and Technology, USA, Tel: (605) 394-1890;

Email: Alevtina.Smirnova@sdsmt.edu

\begin{abstract}
The article is focused on the inelastic deformations of rocks and their influence on the development of oil and gas deposits. The influence of the relaxation deformation of rocks on the parameters of the reservoir development, such as reservoir pressure, porosity, and oil saturation has been addressed. The numerical analysis was performed in assumption that the oil-gas deposits are developed as a uniform grid of wells and under conditions when gas-oil interaction is constant. The performed analysis demonstrates the process of the rock relaxation in regard to the defined specific parameters during the development of the oil-gas deposits. It has been concluded that the rock relaxation has the maximum effect on the oil-gas deposits when the initial reservoir pressure is the highest.
\end{abstract}

Keywords: Oil and gas deposits; Inelastic rock deformation; Relaxation rock deformation; Creeping rock deformation

\section{Introduction}

Oil and gas fields in the subsoil of Earth are present in a deformable state especially in the case of deep fields that exist under high geostatic pressures and are exposed to strong deformations in the course of their development. In the past, these deformations of rocks characterized by a nonlinear behaviour have been extensively investigated [1-12] and allowed to predict the corresponding impacts of deformation processes in oil and gas fields. The numeric study of inelastic deformations under different conditions in porous rocks during different stages of their development and under non-equiaxial loading [11] has been performed. The results of this work demonstrate that the evolution of the yield surface with deformation has major influence on the resulting characteristics of deformation patterns. Furthermore, shear-enhanced compaction and strain localization in case of inelastic deformation [12] has been studied in case of the strain hardening behaviour in the sandstone samples under drainage conditions.

Based on comprehensive study on deformation of reservoir rock and multiphase flow in porous media a three-dimensional and three phase-flow model for prediction of the porosity and permeability changes was 


\section{Petroleum \& Petrochemical Engineering Journal}

created [8] using a finite-difference method and selfadaptive iterative approach. This study implies that the deformation of oil formation may be elastic, elasticplastic, or plastic within the actual oil formation process and during the oil exploitation.

Besides the noted features of the rock deformation, other types of deformation, specifically relaxation and creeping take place. It has been shown that at sharp and considerable changes of pressure the porosity of the rocks has a relaxation behavior [5] that vary from several hours to several years. It was demonstrated that at long-term influence of geostatic pressure the creeping deformation possesses a hereditary property.

Furthermore, it was demonstrated $[9,10]$ that the specific rheological inelastic deformations of rocks have significant impact on the oil/gas fields development, among them creeping deformation of rocks provide the greatest influence.

The goal of this study is in evaluation of the influence of the inelastic deformation of rocks on the development of oil-gas deposits. Compared to the earlier approaches performed for either oil or gas fields separately, the novelty of this study is in characterization of the coherent nature of both oil and gas phases trapped in various ways in sedimentary rock layers. This approach allows to evaluate the deformation processes in the realistic conditions and consider variations for establishing the influence of rheological deformation of rocks on the characteristics of the development of oil-gas fields.

\section{Mathematical Basis for the Modelling Approach}

\section{Filtration of Fluids in the Layers during Relaxation Deformation of Rocks}

The processes of water filtration in friable rocks indicate that such rocks are often deformed. It was demonstrated, that the deformed friable rocks and clay deposits are not elastic, and should be characterized by using a complex rheological approach.

The actual nonlinear dependence of porosity and permeability of rocks on an average normal tension is approximated as a linear or piecewise-linear deformation. However, it can be different during the loading process when the average normal tension $(\sigma)$ is increased and in the process of unloading $\sigma$ reduction takes place. Under loading conditions when decrease in reservoir pressure takes place, the rock is deformed with compressibility $\beta_{c l}$. Under unloading conditions the compressibility is $\beta_{c u}$ and $\beta_{c l}>\beta_{c u}$ :

$$
\beta_{c l}=\left(\frac{\partial m}{\partial \sigma}\right)_{l}, \beta_{c u}=\left(\frac{\partial m}{\partial \sigma}\right)_{u}
$$

where "l" corresponds to the rock loading, and " $u$ " - to the rock unloading.

The approach of the piecewise and linear deformation of rocks when loading and unloading of the rock take place in case of the elasto-plastic mode leads to the solution of the following system of the linear differential equations of the elastic mode:

$$
\begin{gathered}
\frac{\partial p}{\partial t}=\chi_{l} \nabla^{2} p \text { at } \frac{\partial p}{\partial t} \leq 0 \text { (2) } \\
\frac{\partial p}{\partial t}=\chi_{u} \nabla^{2} p \text { at } \frac{\partial p}{\partial t} \geq 0 \\
\chi_{l}=\frac{k}{\mu\left(m \beta_{l}+\beta_{c l}\right)}, \chi_{u}=\frac{k}{\mu\left(m \beta_{l}+\beta_{c u}\right)}
\end{gathered}
$$

where $p$ - reservoir pressure; $m$ - porosity of a layer; $k$ permeability of a layer; $\beta_{l}$ - compressibility of a liquid; $\mu$ - liquid viscosity.

However, in many cases the piecewise and linear deformation approach does not describe the filtration mechanism in the deposits which deform under complicated rheological conditions. It is necessary to consider that the deformed deposit does not come to an equilibrium state instantly.

Based on the assumption of the rheological mode of oil layers, a theory of deposit filtration has been developed based on Maxwell's medium deformation approach. It was accepted that between the porosity and the pressure in the layer the following relationship is valid:

$$
\frac{\partial m}{\partial t}=\beta_{c} \frac{\partial p}{\partial t}+\frac{p-p_{0}}{\mu_{r}}
$$

where $p_{0}$ - initial pressure; $\beta_{c}$ and $\mu_{r}$ - elastic compressibility of the porous environment and viscosity of rocks, respectively. 


\section{Petroleum \& Petrochemical Engineering Journal}

The equation (4) in asymptotic case turns into the law of elastic deformation. So, at $\mu_{m} \rightarrow \infty$ from (4) the following equation can be derived:

$$
\frac{\partial m}{\partial t}=\beta_{c} \frac{\partial p}{\partial t} \text { or } m=m_{0}\left[1+\beta_{r}\left(p-p_{0}\right)\right]
$$

where $m_{0}$ - initial porosity of a layer; $\beta_{r}=\frac{\beta_{c}}{m_{0}}$ compressibility of a rock.

Further, the theory of a filtration in relaxation environment has been developed [5]. In this case, a more general relaxation relationship between the porosity and the pressure of the layer has been derived:

$$
m-m_{0}+\tau_{m} \frac{\partial\left(m-m_{0}\right)}{\partial t}=\beta_{c}\left[\left(p-p_{0}\right)+\tau_{p} \frac{\partial\left(p-p_{0}\right)}{\partial t}\right]
$$

where $\tau_{m}$ - porosity relaxation time; $\tau_{p}$ - pressure relaxation time.

In case of $\tau_{p}=0$, from (6) the following equation can be derived:

$$
m+\tau_{m} \frac{\partial m}{\partial t}=m_{0}\left[1+\beta_{r}\left(p-p_{0}\right)\right](7)
$$

In integrated form it turns into the following relationship:

$$
m=m_{0} e^{-\frac{t}{\tau_{m}}}+\frac{m_{0}}{\tau_{m}} \int_{0}^{t} \exp \left[-\frac{t-\tau}{\tau_{m}}\right]\left[1+\beta_{r}\left(p-p_{0}\right)\right] d \tau
$$

Instead of (7) and (8), a more complicated law of porosity relaxation has been used [5,9]:

$$
m+\tau_{m} \frac{\partial m}{\partial t}=m_{0} \exp \left[\beta_{r}\left(p-p_{0}\right)\right](9)
$$

In integrated form it gives the following equation:

$$
m=m_{0} e^{-\frac{t}{\tau_{m}}}+\frac{m_{0}}{\tau_{m}} \int_{0}^{t} \exp \left[-\frac{t-\tau}{\tau_{m}}\right] \exp \left[\beta_{r}\left(p-p_{0}\right)\right] d \tau
$$

From (7) and (9) in case of $\tau_{m}=0$, the equations of porosity in linear and nonlinear elastic media can be derived. The laws for the speed and porosity relaxation of the layers result in a following equation of a linear relaxation of filtration:

$$
\bar{v}+\tau_{q} \frac{\partial \bar{v}}{\partial t}=-\frac{k}{\mu} \nabla\left(p+\tau p \frac{\partial p}{\partial t}\right)
$$

where $\tau_{q}$ - time of a relaxation of the speed (output) and

$$
\tau_{q}<\tau_{p}
$$

It is necessary to note that Equation 11 allows for special cases, for example at $\tau_{q}=\tau_{p}=0$ Equation11 turns into the Darcy's law. Substitution in Equation11 of the continuity and in consideration of a linear dependence of porosity, permeability, and density, a differential equation of the relaxation filtration of liquid can be derived:

$$
\nabla^{2}\left(p+\tau_{p} \frac{\partial p}{\partial t}\right)=\frac{1}{\chi} \frac{\partial}{\partial t}\left(p+\tau_{q} \frac{\partial p}{\partial t}\right)
$$

This equation shows that dependence of porosity on pressure reaches equilibrium only at small pressure changes. However, at large pressure changes this dependence has a relaxation character which needs to be considered for all dynamic parameters of fluids in the layer.

Further, the liquid filtration in the porous environment presented by the Darcy's law takes into account porosity relaxation under the law (7). In this case, an equation is similar to Equation12:

$$
\nabla^{2}\left(p+\tau_{m} \frac{\partial p}{\partial t}\right)=\frac{1}{\chi} \frac{\partial}{\partial t}\left(p+\tau_{1} \frac{\partial p}{\partial t}\right)
$$

where $\tau_{1}=\tau_{m}\left(1-\frac{\beta_{c}}{\beta^{*}}\right) ; \beta^{*}=m_{0} \beta_{l}+\beta_{c}$.

\section{Filtration of Fluids in the Layers at Creeping Deformation of the Rocks}

It has been demonstrated [5], that a general relaxation behavior between porosity and internal reservoir pressure is not accurate. This deformation dependence of rocks should consider heredity that requires a description of deformation processes in rocks and other rheological interrelations specific to the creep of materials. 


\section{Petroleum \& Petrochemical Engineering Journal}

The hydrodynamic data for the wells of many deposits confirm that the actual time of full stabilization of reservoir pressures can be over several months. At the same time the theory of the elastic mode shows that the estimated time of this stabilization is much smaller. This can be due to creeping deformations of the porous environment, which in turn is directly related to the longterm exposure to geostatic pressures.

The mathematical description of the relaxation phenomena for liquid and gas filtration in the layers with creeping deformation has been used [5] considering the volume creeping of rocks. In this case, permeability and porosity of rocks is described by the following equations:

$$
\begin{array}{r}
k=k_{0}\left[1+k_{1} \int_{0}^{t} e^{-\frac{t-\tau}{\tau_{k}}}\left(p-p_{0}\right) d \tau\right] \\
m=m_{0}\left[1+m_{1} \int_{0}^{t} e^{-\frac{t-\tau}{\tau_{m}}}\left(p-p_{0}\right) d \tau\right]
\end{array}
$$

where $k_{0}$ - initial permeability of layer; $m_{1}, k_{1}$ - creeping parameters ( $m_{1}=1 / \mu$ - volume fluidity); $\tau_{k}$ and $\tau_{m}$ time of a relaxation of permeability and porosity of the creeping environment, respectively.

It is necessary to note, that in (14) and (15) the fading exponential function was used. Similar dependences of permeability (14) and porosity (15) can be received using other functions of a creep core. For example, for Abel kernel:

$$
\begin{aligned}
& k=k_{0}\left[1+\delta_{k} \int_{0}^{t}(t-\tau)^{-\alpha}\left(p-p_{0}\right) d \tau\right] \\
& m=m_{0}\left[1+\delta_{m} \int_{0}^{t}(t-\tau)^{-\alpha}\left(p-p_{0}\right) d \tau\right]
\end{aligned}
$$

where $\delta_{k}, \delta_{m}, \alpha$ - creeping parameters, and $0<\alpha<1$.

The general dependence of the description of permeability and the porosity differing from (14)-(15) $[5,9]$ in case of Volterra kernel:

$$
\begin{aligned}
& k=k_{0}\left[1+\alpha_{k}\left(p-p_{0}\right)+k_{1} \int_{0}^{t} e^{-\frac{t-\tau}{\tau_{k}}}\left(p-p_{0}\right) d \tau\right] \\
& m=m_{0}\left[1+\beta_{r}\left(p-p_{0}\right)+m_{1} \int_{0}^{t} e^{-\frac{t-\tau}{\tau_{m}}}\left(p-p_{0}\right) d \tau\right]
\end{aligned}
$$

where $\alpha_{k}$ and $\beta_{n}$ - coefficients of elastic change of permeability and elastic compressibility of the environment, respectively.

\section{Computational Results and Discussion}

While solving the problem of the influence of inelastic deformations of rocks on the parameters of the oil-gas deposits, an assumption is made that the deposit is developed by a uniform grid of wells under condition of a constant gas-oil contact (GOC). This is possible by considering that despite the fact that both oil and gas are the parts of the same deposit, the position of gas-oil contact during this process does not change. The constant GOC of gas-oil contact during the oil extraction is provided by the rate of gas production from a gas cap with time. It is assumed that the oil part of the deposit is developed at a mode of the dissolved gas and the rocks are deformed with relaxation.

The model addressing these issues has been derived from the mass balance equation for the oil and gas and the law of porosity relaxation. By using the mass balance equation, it is justified that the deposit can be developed by a uniform grid of wells when the energy of a reservoir system within regular time intervals is evenly distributed within the whole volume of the deposit.

The equations of mass balance for oil and gas can be written as following:

$$
\bar{q}_{o}=-\frac{d}{d t}\left[\frac{\sigma \bar{m}}{\bar{a}(P)}\right]
$$

$$
\begin{gathered}
\bar{q}_{g o} P_{a t m}=-\frac{d}{d t}\left[(1-\sigma) a\left(P_{0}\right) \bar{m} P+\frac{\sigma \bar{m}}{\bar{a}(P)} \frac{S(P)}{\beta}\right] \beta \\
\bar{q}_{g g}=-\frac{d}{d t}\left[\frac{\bar{m} P}{\bar{z}(P) P_{0}}\right]
\end{gathered}
$$




\section{Petroleum \& Petrochemical Engineering Journal}

where $\bar{q}_{o}=\frac{q_{o}}{V_{o}} ; \bar{q}_{g o}=\frac{q_{g o}}{V_{o}} ; \bar{q}_{g g}=\frac{q_{g g}}{V_{g}} ; \bar{m}=\frac{m}{m_{0}} ;$ $\bar{a}(P)=\frac{a(P)}{a\left(P_{0}\right)} ; \bar{z}(P)=\frac{z(P)}{z\left(P_{0}\right)} ; q_{o}$ - extraction of oil from a deposit (units of time); $q_{g g}, q_{g o}$ - extraction of gas, from a gas cap and oil area, respectively; $P_{0}, P$ pressure of initial and current average weighted reservior; $m_{0}, m$ - mean porosity, corresponding pressure $P_{0}$ and $P ; \sigma$ - average oil-saturation within the limits of an oil part of a deposit; $a(P)$ - volumetric factor of oil; $S(P)$ - solubility of gas in oil; $z(P)$ - gas compressibility factor; $V_{o}$ - volume of oil reservoir; $V_{g}$ volume of gas reservoir; $\beta$ - temperature amendment for gas; $P_{a t}$ - atmospheric pressure; $t$ - time of development.

Taking into account the rock relaxation, the average porosity is determined from the equation:

$$
\bar{m}+\tau_{m} \frac{d \bar{m}}{d t}=\exp \left[\beta_{r}\left(P-P_{0}\right)\right]
$$

The equations (20), (21) and (23) allow to define the time change of the average values of the reservoir pressure $(P)$, oil saturation $(\sigma)$, and porosity $(m)$ in an oil part of the deposit.

To determine the unknown quantities of $P, \sigma$ and $m$ by solving the mass balance equations for oil and gas and Equation23, the following system of differential equations can be obtained:

$$
\left\{\begin{array}{l}
\frac{d P}{d t}=f_{P}(t, \bar{m}, \sigma, P) \\
\frac{d \sigma_{1}}{d t}=f_{\sigma_{1}}(t, \bar{m}, \sigma, P) \\
\frac{d \bar{m}}{d t}=f_{\bar{m}}(\bar{m}, P)
\end{array}\right.
$$

where $f_{P}=-\frac{\overline{q_{o}}[\varphi(P)+\overline{G F}]+a\left(P_{0}\right) P f_{\bar{m}}}{\bar{m}\left[a\left(P_{0}\right)-\sigma_{1} \varphi^{\prime}(P)\right]}$;

$$
\begin{gathered}
f_{\bar{m}}=\frac{1}{\bar{m}}\left\{\exp \left[\beta_{r}\left(P-P_{0}\right)\right]-\bar{m}\right\} ; \\
f_{\sigma_{1}}=-\frac{1}{\bar{m}}\left[\overline{q_{o}}(t)+\sigma_{1} f_{\bar{m}}\right] ; \sigma_{1}=\frac{\sigma}{\bar{a}(P)} ; \\
\varphi(P)=P a(P)-\frac{S(P)}{\beta} ; \\
\overline{G F}=\frac{G F \cdot P_{a t}}{\beta} ;
\end{gathered}
$$

and $G F$ is the gas-oil ratio.

The resulting system was solved by the Runge-Kutta method [13] with the following initial conditions:

at $t=0-P=P_{0} ; \sigma=1 ; \bar{m}=1$.

In the case of deposits with non-linear elastically deformable medium $\left(\tau_{m}=0\right)$ and for $f_{P}$ and $f_{\bar{m}}$ the values are as follows:

$$
\begin{gathered}
f_{P}=-\frac{\bar{q}_{o}[\varphi(P)+\overline{G F}]}{\bar{m}\left[a\left(P_{0}\right)+\beta_{r} a\left(P_{0}\right) P-\sigma_{1} \varphi^{\prime}(P)\right]} ; \\
f_{\bar{m}}=\beta_{r} \bar{m} f_{P}(26)
\end{gathered}
$$

The extraction of gas from a gas cap in assumption of constant GOC can be defined from Equation27 and by differentiation of the right part of Equation22:

$$
\bar{q}_{g g}=-\left\{\frac{P}{\bar{z}(P) P_{0}} f_{\bar{m}}+\left[\frac{\bar{m}}{\bar{z}(P) P_{0}}-\frac{z^{\prime}(P) \bar{m} P}{\bar{z}(P) P_{0}}\right] f_{P}\right\}
$$

where $f_{\bar{m}}$ and $f_{P}$ are defined according to the pressure and porosity of the differential expressions. The determined dynamics of gas extraction from the gas part of the deposit ensures the preservation of the constant GOC values in the process of oil/gas extraction.

The dynamics of a gas production from all deposits ( $q_{g}$ ) is defined from the following expression:

$$
q_{g}=q_{g g}+G F \cdot q_{o}
$$




\section{Petroleum \& Petrochemical Engineering Journal}

The specified equation defines the basic parameters of the oil/gas deposit defined by a uniform grid of wells at constant GOC in presence of relaxation.

The effect of the rock relaxation time on the parameters of the oil/gas deposit (reservoir pressure, oil saturation, porosity) was evaluated considering that the oil recovery constant $q_{o}=2000 \mathrm{~m}^{3} /$ day, initial reservoir pressure $P_{o}=40$ or $60 \mathrm{MPa}$, and the time of relaxation $\tau_{m}=5$ years (Figure 1 and Figure 2). The results were compared to the corresponding data obtained in a nonlinear elastic deformable environment ( $\tau_{m}=0$ ).

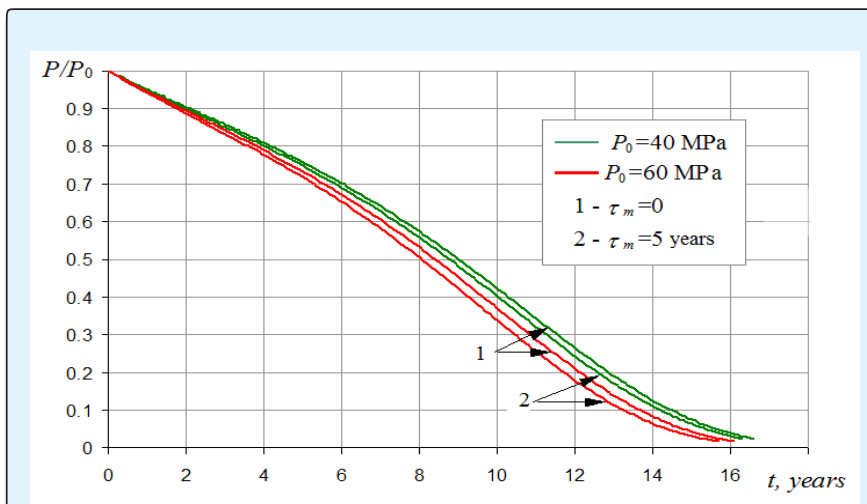

Figure 1: Change in time reservoir pressure within the oil/gas reservoir vs. time of rock relaxation at different initial reservoir pressures.

It can be seen from Figure 1 that the influence of the relaxation deformation of rocks on the change in reservoir pressure during the initial development period (up to about 2 years) is insignificant, and in the subsequent moments it gradually increases. This may be due to the fact that during this period of development, both in the case of nonlinear elastic and in the case of relaxation deformation of reservoir rocks, the porosity of the formation was reduced in a small interval relative to the initial value, and this resulted in an insignificant difference between the corresponding values of the reservoir pressure. Since the influence of the relaxation deformation of a porous medium with respect to the nonlinear - elastic deformation of the formation does not disappear until the end of the deposit development (Figure 2), its influence on the determined development indices, accordingly, manifests itself in the main for the whole period of reservoirs development, some marked initial period of its natural exhaustion. It should be noted that this kind of change in the development indices of the deposit was also observed for oil saturation.

The results also indicate that the reservoir pressure (and oil saturation) within the deposit in presence of relaxation decreases faster than in presence of nonlinear elastic and deformable environment, and on the contrary the porosity within the deposit in presence of relaxation decreases slowly than in presence of nonlinear elastic and deformable environment (Figure 1 and Figure 2).

Based on the proposed model also it was confirmed that in the deposits with high initial reservoir pressure (e.g. $60 \mathrm{MPa}$ ) differences between the corresponding values in time of reservoir pressure and porosity for relaxation deformable environment are higher than for nonlinear elastic deformable environment $\left(\tau_{m}=0\right.$ ).

The main conclusions following from the received results of calculations are given in the following item of article

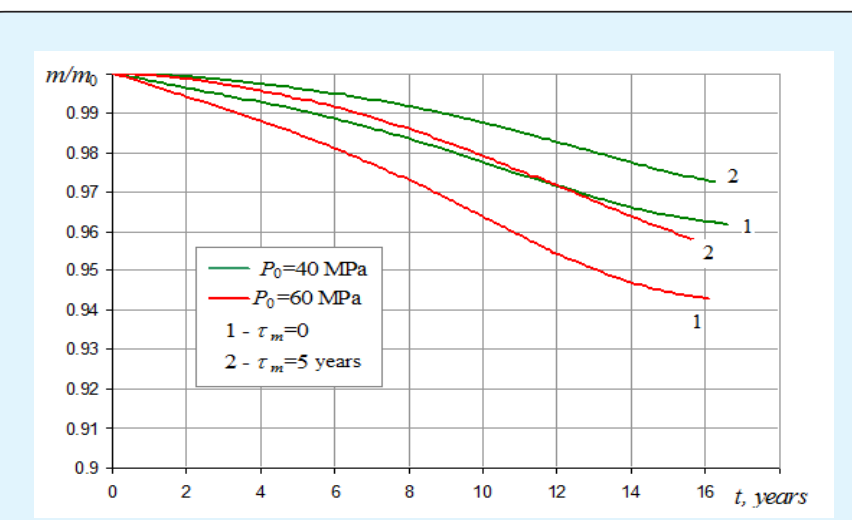

Figure 2: Change in porosity within the oil/gas reservoir vs. time of rock relaxation at different initial reservoir pressures.

\section{Conclusions}

The results obtained in this study indicate that the most significant effect is related to the reduction of the relaxation time related to the porosity of the deposit. In deposits with nonlinear elastic deformations the porosity decreases faster than in deposits within higher relaxation environment. Decrease of porosity slows down with increase in the time of a rock relaxation.

In deposits with nonlinear elastic deformations the reservoir pressure (oil-saturation) decreases slowly than 


\section{Petroleum \& Petrochemical Engineering Journal}

in deposits within higher relaxation environment. Decrease of reservoir pressure (oil-saturation) slows down with reduction in the time of a rock relaxation.

At the initial stage, the pressure decrease (oilsaturation) in deposits with nonlinear elastic deformable environment and with relaxation occurs equally fast. However, with time and compared to the deposits with relaxation environment, the process of a nonlinear elastic deformation corresponds to the slower pressure decrease.

Defined by the presence of gas in oil/gas reservoir, pressure growth results in a noticeable difference between the pressure with relaxation ( $\tau_{m}=5$ years) and nonlinear elastic deformation $\left(\tau_{m}=0\right)$. In this case the deformable environment becomes more important. Thus, the growth of the initial reservoir pressure highlights the effect of rock relaxation on reservoir pressure and oil saturation.

It can be concluded that gas production for preservation of the gas-oil contact in the deposits with relaxation environment is more important than in deposits with nonlinear elastic deformations. However, this importance is more pronounced in the case of deposits with high initial reservoir pressure.

Considering the rock relaxation, the parameters of the oil/gas deposits have the highest values at the high initial reservoir pressure. Specifically, the basic parameters of the deep-laying oil/gas deposits at high initial reservoir pressure are necessary to consider due to highly pronounced effects of rock relaxation.

\section{Acknowledgements}

The authors gratefully acknowledge the help from the Director of Oil and Gas Institute of the Azerbaijan National Academy of Sciences, Academician Dr. İbrahim Guliyev during preparation of this manuscript.

\section{References}

1. Ranalli G (1995) Rheology of the Earth. 2nd(Edn.), Springer Netherlands, pp: 1-414.

2. Cristescu ND (2002) New trends in rock mechanics. Int Appl Mech 38(1): 1-22.
3. Jing L, Hudson JA (2002) Numerical methods in rock mechanics. Int J of Rock Mech Min Sci 39(4): 409-427.

4. Antman SS (2005) Nonlinear Problems of Elasticity. $2^{\text {nd }}(E d n)$, Springer-Verlag New York, pp: 838.

5. Guliyev AM, Kazymov BZ (2009) Deformation of rocks and its influence on their filtrations-capacitor properties and on processes of a filtration and development of oil and gas deposits. Baku.

6. Jones RM (2009) Deformation theory of plasticity. Bull Ridge Corporation, Blacksburg.

7. Dobrynin VM, Gorodnov AV, Chemoglazov VN, Davydova OP (2012) Technique of the assessment of irreversible deformations and change of the tension of breeds in the course of development of fields of hydrocarbons and operation of underground gas storages. Logger 4: 59-68.

8. Ju B (2014) Mathematical model and numerical simulation of multiphase-flow in permeable rocks considering diverse deformation. J Pet Sci Eng 119: 149-155.

9. Guliyev AM, Kazymov BZ (2012) Study of rhealogical properties of reservoirs during relaxation and creep strain of rocks and usage of these properties for addressing of applied objectives of oil-gas fields development. Special issue of $34^{\text {th }}$ International Geological Congress "The modern problems of geology and geophysics of Eastern Caucasus and the South Caspian depression".

10. Kazymov BZ (2017) Numerical definition of indicators of the development of creeping oil layer on values of change of an well production. Int J Theory Appl Math 3(5): 167-173.

11. Stefanov YP, Chertov MA, Aidagulov GR, Myasnikov AN (2011) Dynamics of inelastic deformation of porous rocks and formation of localized compaction zones studied by numerical modeling. J Mech Phys Solids 59(11): 2323-2340.

12. Baud P, Vajdova V, Wong T (2006) Shear-enhanced compaction and strain localization: inelastic deformation and constitutive modeling of four porous sandstones. J Geophys Res 111(B12).

13. Delin T, Zheng Ch (2012) On A General Formula of Fourth Order Runge-Kutta Method. J Math Sci Math Edu 7(2): 1-10. 


\section{Petroleum \& Petrochemical Engineering Journal}

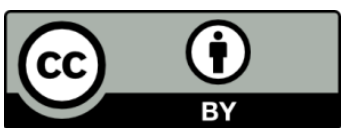

